\title{
Responsabilité sociale : un point de vue philosophique
}

\section{Social accountability: a philosophical perspective}

\author{
Céline LEFÈVE ${ }^{1}$, Valérie GATEAU ${ }^{2}$ \\ 1 Maître de conférences en philosophie de la médecine, Centre Georges Canguilhem/SPHERE UMR 7219, Université \\ Paris Diderot, France \\ 2 Post-doctorante en éthique médicale, Centre Georges Canguilhem/Hôpital Beaujon/Programme interdisciplinaire \\ Université Sorbonne Paris Cité « La Personne en médecine », Université Paris Diderot, France
}

Manuscrit reçu le 17 novembre 2015 ; commentaires éditoriaux formulés aux auteurs le 7 janvier 2016 ; accepté pour publication le 20 janvier 2016

\section{Mots-clés \\ Responsabilité sociale ; Ethique ; \\ Sciences humaines et sociales ; \\ formation médicale}

\section{Keywords} Social accountability; Ethics; Human and social sciences; Medical education

\begin{abstract}
Résumé - Problématique : L'article interroge la nouveauté historique du concept de responsabilité sociale de la médecine, en faisant retour sur l'histoire de la médecine sociale, ainsi que sa nouveauté éthique, en rappelant qu'il fait partie de l'identité professionnelle médicale. Exégèse : Les auteurs montrent que ce concept implique de se demander comment définir démocratiquement les politiques de santé en général, et les programmes de formation médicale en particulier. Ils affirment que les recherches et enseignements en sciences humaines et sociales jouent un rôle précieux pour connaître l'évolution des besoins, des valeurs et des services en santé.

Abstract - Context: The paper challenges the historical novelty of the social-responsibilityof-medicine concept by reviewing the history of social medicine and its ethical novelty and highlighting that it is part of the medical identity. Analysis: The authors show that this concept involves asking ourselves how we can democratically determine health policies in general and medical education programs in particular. Lastly, they state that research and teaching in the field of human and social sciences play a valuable role in identifying changes in needs, values and health services.
\end{abstract}

Le Consensus mondial sur la responsabilité sociale des facultés de médecine, publié en 2010 et issu de la collaboration de 130 experts internationaux ${ }^{[1]}$, propose dix directions stratégiques pour la formation médicale, en rapport avec la responsabilité sociale tant des facultés de médecine que des futurs praticiens. Ces propositions visent à améliorer les réponses aux futurs défis de santé dans la société (notamment au regard des inégalités sociales de santé, de l'accès des plus vulnérables aux soins primaires, 
des déterminants environnementaux, sociaux et individuels de la santé, etc.). Le Consensus porte une attention particulière aux moyens de mieux implémenter les politiques de santé publique dans la société civile grâce à une formation plus responsable des praticiens. Ce Consensus implique donc de questionner les liens entre médecine et société, ainsi que la place et le rôle que doit y prendre la formation médicale.

\section{La responsabilité sociale de la médecine : innovation ou continuation historique?}

L'idée que la médecine revêt une fonction sociale n'est pas nouvelle, elle peut être située historiquement. Elle apparaît au moins avec la naissance de la médecine sociale au $18^{\text {ème }}$ siècle (attention à la natalité, la mortalité et la morbidité, à la santé des enfants ; mesures de salubrité dans les villes, logements et lieux de travail). Elle trouve son plein accomplissement dans l'hygiénisme du $19^{\text {ème }}$ siècle (éducation sanitaire et stigmatisation des comportements jugés déviants comme la masturbation et l'alcoolisme) puis dans les politiques de santé publique au $20^{\text {ème }}$ siècle (campagnes de vaccinations ; prévention des comportements à risque). On peut donc s'interroger sur le caractère novateur du concept de responsabilité sociale de la médecine et, au-delà, sur les limites et risques des politiques qui s'en inspirent. En effet, ainsi que l'ont souligné Michel Foucault ${ }^{[2]}$ et Georges Canguilhem ${ }^{[3]}$, le gouvernement des vies dans un objectif d'amélioration de la santé publique comporte des risques : normalisation des comportements, stigmatisation des individus, exclusion des plus vulnérables - pourtant censés être les principaux bénéficiaires de ces politiques.

Par ailleurs, l'engagement et la responsabilité des praticiens auprès des patients, des confrères et de l'ensemble de la communauté sont reconnus comme faisant partie de l'identité professionnelle des médecins. Acquérir des habitudes de sentir, de penser et d'agir qui permettent de faire preuve d'une attitude moralement et socialement responsable constitue bien évidemment un objectif essentiel de la formation médicale. C'est d'ailleurs pour ces raisons que la formation initiale comporte des enseignements d'éthique, d'histoire de la médecine, et de sciences sociales $^{[4-6]}$. Sur ce second plan, le concept de responsabilité sociale s'ancre donc dans le socle des valeurs partagées par la profession médicale. Là encore, le concept n'est pas nouveau, mais reformule des enjeux déontologiques évoluant avec la société et les problématiques de santé.

\section{Les défis de la « responsabilité sociale »}

Le concept de responsabilité sociale de la médecine est donc à juste titre reformulé par le Consensus qui insiste sur l'importance des partenariats entre les universités, le système de santé et les acteurs de la société civile pour l'amélioration de la santé. En ce sens, il génère des défis méthodologiques. En amont, la définition de l'orientation des politiques de santé publique interroge les modèles de la délibération publique. Si la volonté de faire participer plus et mieux les représentants de la société civile (associations, représentants des communautés et usagers du système de soins) est affirmée, les moyens de la réaliser restent à définir. Comment organiser la délibération publique dans les choix de santé ? Faut-il avoir recours à des modèles universalistes qui visent un consensus moral au nom de valeurs partagées (comme la protection de la vulnérabilité ou le respect de la dignité, etc.), ce qui comporte le risque d'une éthique abstraite et éloignée des situations particulières? Faut-il au contraire documenter directement les valeurs et préférences des citoyens, notamment par des enquêtes sociologiques ? Ou encore faut-il choisir une délibération politique assortie d'un vote? Ces questions se posent de la même manière pour donner chair au concept de responsabilité sociale dans la formation médicale et déterminer concrètement les contenus, méthodes et disciplines qui doivent être enseignées. Qui en décidera : les responsables politiques, les professionnels de santé, les associations et patients, les étudiants ? Selon quelles modalités ces décisions seront-elles prises ? La responsabilité sociale des facultés de médecine appelle à innover et à expérimenter différents modèles délibératifs. 


\section{Le rôle des sciences humaines et sociales}

On comprend aussi que le développement de recherches en sciences humaines et sociales et leur intégration à la formation médicale tout au long du cursus sont indispensables. Il n'est pas seulement fondamental d'élaborer des modèles délibératifs adaptés en vue de déterminer démocratiquement les politiques de santé auxquelles s'articule la formation. Il est aussi nécessaire de documenter les besoins de santé des populations, l'évolution des valeurs et des demandes sociales en santé, ou encore les transformations des services de santé. On peut à cet égard regretter que le Consensus n'évoque pas la nécessité et l'apport, pour la formation, des recherches en sciences humaines et sociales. On sait, pour ne citer qu'un exemple, que la qualité de la prise en charge des patients dépend des interactions entre des professionnels (généralistes/spécialistes, médecins/paramédicaux, services médico-sociaux, administrations) et des institutions de santé multiples (hôpitaux, réseaux de santé, médecins de ville, établissements spécialisés). De manière générale, on sait que les médecins sont aujourd'hui confrontés - et c'est bien ce que montre le Consensus - à des problèmes complexes qui mêlent des dimensions politiques, économiques, organisationnelles, sociales et éthiques. Or, sur ces différents points, des recherches et des enseignements sur des questions telles que l'inter professionnalité, le partage des informations et des décisions, les orientations économiques du système de santé, la prise en charge des maladies chroniques et du vieillissement, etc. s'avèrent précieux ${ }^{[7]}$.

Il faut se féliciter, nous semble-t-il, de la promotion de la notion de responsabilité sociale qui traduit au moins deux évolutions notables dans les objectifs de la formation médicale. Premièrement, elle montre que celle-ci vise désormais autant la construction de l'identité professionnelle de médecin à travers l'acquisition de savoirs et d'habitus médicaux, que la compréhension et l'adaptation des pratiques de santé aux besoins des populations. Former les médecins viserait donc autant à les intégrer au corps social qu'au corps médical. Deuxièmement, elle montre que les questions d'éthique médicale ne sont plus désormais centrées sur l'évolution des techniques (réanimation, greffe, assistance médicale à la procréation, etc.) mais prennent en charge des questions sociales et politiques globales (risques environnementaux ou médicamenteux, inégalités de santé et d'accès aux soins, migrations, etc.). La notion de responsabilité sociale traduit sans aucun doute un dépassement de l'éthique du colloque singulier vers une philosophie du soin tant sociale qu'environnementale.

\section{Contributions}

Céline Lefève et Valérie Gateau ont co-rédigé solidairement le manuscrit.

\section{Déclaration d'intérêts}

Aucune des auteures ne déclare de conflit d'intérêt en lien avec le contenu de cet article.

\section{Approbation éthique}

Sans objet, non sollicitée

\section{Références}

1. Contribution collective de la conférence pour le consensus mondial sur la responsabilité sociale des facultés de médecine. Consensus mondial sur la responsabilité sociale des facultés de médecine. Pédagogie Médicale 2011;12:37-48.

2. Foucault M. « Crise de la médecine ou crise de l'antimédecine » (1976), in Dits et écrits II, $\mathrm{n}^{\circ} 170$, Paris : Gallimard, coll. Quarto, 1994, p. 40-58 ; « La naissance de la médecine sociale » (1977), in Dits et écrits II, $\mathrm{n}^{\circ}$ 196, Paris : Gallimard, coll. Quarto, 1994, p. 207-228 ; « Naissance de la biopolitique » (1979), in Dits et écrits II, $\mathrm{n}^{\circ} 274$, Paris : Gallimard, coll. Quarto, 1994, p. 818-826. 
3. Canguilhem G. Ecrits sur la médecine, Paris : Seuil, 2002.

4. Boch AL. L'enseignement de l'éthique au cours des premier et deuxième cycle des études médicales. Etat des lieux dans les facultés de médecine françaises Ethique et santé 2010;7:117-122

5. Lefève C. L'enseignement de l'éthique dans les études médicales en France. Etat des lieux et présentation d'un exemple d'enseignement. In C. Byk (dir). La formation à l'éthique des scientifiques. Bordeaux : Les Etudes hospitalières, 2014:57-77
6. Gaillard M, Lechopier N. Relever le défi d'introduire aux sciences humaines et sociales en première année commune des études de santé. Mise en perspective de quelques pratiques pédagogiques. Pédagogie Médicale 2015;16:23-34.

7. Lefève C, Mino J-C. Former de vrais thérapeutes. La place des sciences humaines et sociales dans les études médicales. Etudes 2011;414:187-98.

Correspondance et offprints: Céline Lefève, Centre Georges Canguilhem/SPHERE UMR 7219, Université Paris Diderot, France. Mailto : celine.lefeve@gmail.com 